\section{Triglyceride trouble from tumors}

\section{By Lev Osherovich, Senior Writer}

A team led by researchers at the University of South Florida has found that cancer cells alter both the amount and the type of lipid in dendritic cells, decreasing their ability to initiate immunity. The finding could explain the poor immune response seen in many cancer patients - a phenomenon that has confounded many efforts to develop vaccines against tumor antigens.

The team also included researchers from the H. Lee Moffitt Cancer Center \& Research Institute, Imperial College London and the University of Pittsburgh.

Because dendritic cells (DCs) collect antigens and present them to $\mathrm{T}$ helper cells, they already are the targets of cancer vaccines and adjuvants. For example, Dendreon Corp.'s Provenge sipuleucel-T, the first cancer vaccine therapy approved in the U.S., is created by delivering a prostate cancer antigen to cultured DCs and then returning the cells to the patient's body.

The team's findings could help explain why ex vivo strategies like Provenge have succeeded and direct in vivo vaccination with tumor antigens has failed. The study also suggests that restoring normal lipid content in DCs could improve the efficacy of cancer vaccines in development.

"It's been known for years that the immune system in cancer patients isn't working normally," said Dmitry Gabrilovich, professor of oncologic sciences and molecular medicine at South Florida and senior author of the paper in Nature Medicine describing the findings. " "One of the reasons for this is that dendritic cells are not functional. Dendritic cells can be detected in patients with cancer, but they're not performing their normal job of taking up antigens and stimulating T cells."

Gabrilovich drew on prior evidence that certain dietary lipids could inhibit DC activity in vitro ${ }^{2}$ and hypothesized that DCs exposed to tumors have altered lipid levels. Indeed, his team found that DCs from tumor-bearing mice and from cancer patients had greater membrane lipid content than DCs from healthy controls.

The Gabrilovich group then drilled down and examined the relative levels of various membrane lipids in DCs from healthy and tumor-bearing mice and found that triglycerides comprise the most overabundant lipid in the tumor-associated DCs. This was caused by excessive absorption of triglycerides by tumor-exposed DCs.

His team found that the consequence of carrying excess triglyceride baggage is impaired DC function. DCs isolated from tumor-bearing mice were less effective at processing and presenting antigens and eliciting a T cell response ex vivo than DCs from healthy mice.

Likewise, a model antigen injected into tumor-bearing mice underwent DC-mediated processing more slowly than in healthy mice, indicating the defect applied to other antigens besides those coming from tumors.

\section{Lipid-lowering strategy}

Because preventing lipid dysregulation in DCs could increase immune response to tumor antigens, Gabrilovich's findings set the stage for the hunt for new targets that affect lipid levels in DCs.

"We've known that tumors can inhibit the immune system, but this is a new mechanism," said Dendreon SVP, CSO and director David Urdal. "This paper adds to the list of things we're looking at to enhance the immune response we see with Provenge."

Gabrilovich thinks that lowering overall triglyceride synthesis in the body could do the trick. His team found that tumor-bearing mice treated with a research compound that blocks fatty acid synthesis had a more vigorous DC response than tumor-bearing mice treated with solvent. When vaccinated with a tumor antigen, the mice previously treated with the research compound launched a more robust immune response and had smaller tumors than similarly treated mice with normal fatty acid levels.

An alternative strategy would be to directly block triglyceride transporters that import the extra triglycerides. The Florida-led team found that DC lipid levels could be partially normalized by immunodepletion or genetic ablation of macrophage scavenger receptor 1 (MSR-1; SR-A; CD204), a lipid transporter that is upregulated in tumor-associated DCs.

However, antagonizing MSR-1 might have unwanted effects on normal triglyceride metabolism in other tissues, according to Urdal. "If you wanted to target the scavenger receptor, you would first look at the distribution of that receptor to see if there's any toxicity," he said.

Gabrilovich said his team is now running genetic and pharmacological screens to identify both the tumor-derived factors that are responsible for raising triglyceride levels and compounds that prevent triglyceride accumulation in DCs. He did not patent his findings.

\section{In vivo or ex vivo?}

Until a specific inhibitor of triglyceride uptake is identified, the easiest way to avoid the dampening effect of excess lipids on DCs may be to culture them ex vivo, as Dendreon and others do.

Dendreon has a second cancer vaccine, Neuvenge lapuleucel-T, in Phase I testing for breast and ovarian cancers. Neuvenge will start Phase II testing for bladder cancer by the end of the year, Urdal told SciBX.

Likewise, Argos Therapeutics Inc.'s cancer vaccination technique involves ex vivo activation of DCs. AGS-003 immunotherapy is in Phase II testing for renal cancer in partnership with Kirin Brewery Co. Ltd.

Charles Nicolette, CSO and VP of R\&D at Argos, said the company's platform involves isolating a patient's monocytes, inducing 


\section{ANALYSIS}

their differentiation into DCs ex vivo and then exposing the DCs to a "boatload of tumor antigens made from RNA isolated from tumors."

The resulting DCs are never exposed to the tumor environment that leads to triglyceride buildup, Nicolette noted.

This study "serves as a cautionary tale for people who are attempting to administer antigens directly into a patient," said Nicolette. "They're trying to leverage damaged machinery."

Tibor Keler, founder, SVP and CSO of Celldex Therapeutics Inc., said Gabrilovich's study "makes a good case that animals and humans with tumors have a subset of dendritic cells with higher lipid content than normal and that this is likely to impact their function. There are many ways that tumors subvert the immune response, and this could be one of the important ones."

Celldex's cancer vaccine platform delivers tumor antigens to DCs in vivo with the aid of a DC surface receptor-targeting $\mathrm{mAb}$. The company's CDX-1307, a mAb-linked tumor antigen, is in Phase II testing for bladder cancer.

"It would be interesting to look at lipid levels in our preclinical models," said Keler. "We are targeting DCs that, according to these investigators, would be less competent than normal."

In defense of in vivo targeting, Keler noted that ex vivo DC priming approaches do not fully recapitulate the complex environment of the lymphatic system where DCs interact with T cells to kick off acquired immunity.
He said Celldex's method of delivering an antigen to a large number of DCs in vivo "can overcome the low number and low avidity of precursor T cells" that recognize tumor antigens, which are typically self-proteins that the immune system ordinarily tries to ignore.

Keler, Urdal and Nicolette all noted that next-generation cancer vaccines could employ a combination of lipid-lowering agents, such as toll-like receptor agonists or mAbs that activate DC surface proteins, as part of a package of adjuvants to increase DC activation.

Osherovich, L. SciBX 3(29); doi:10.1038/scibx.2010.881

Published online July 29, 2010

\section{REFERENCES}

1. Herber, D.L. et al. Nat. Med.; published online July 11, 2010; doi:10.1038/nm.2172

Contact: Dmitry I. Gabrilovich, H. Lee Moffitt Cancer Center \& Research Institute, Tampa, Fla.

e-mail: dmitry.gabrilovich@moffitt.org

2. Zeyda, M. et al. J. Biol. Chem. 280,14293-14301 (2005)

\section{COMPANIES AND INSTITUTIONS MENTIONED}

Argos Therapeutics Inc., Durham, N.C.

Celldex Therapeutics Inc. (NASDAQ:CLDX), Needham, Mass.

Dendreon Corp. (NASDAQ:DNDN), Seattle, Wash.

H. Lee Moffitt Cancer Center \& Research Institute, Tampa, Fla. Imperial College London, London, U.K.

Kirin Brewery Co. Ltd. (Tokyo:2503; Pink:KNBWY), Tokyo, Japan

University of Pittsburgh, Pittsburgh, $\mathrm{Pa}$.

University of South Florida, Tampa, Fla. 